\title{
The Hilbert Transform and Fine Continuity
}

\author{
J. B. TWOMEY
}

\begin{abstract}
It is shown that the Hilbert transform of a function having bounded variation in a finite interval $[c, d]$ has fine continuity properties at points in $[c, d]$ outside certain exceptional sets.
\end{abstract}

\section{INTRODUCTION}

The Hilbert transform of a function $f \in L(\mathbb{R})$ is defined by

$$
\mathcal{H} f(x)=\lim _{\varepsilon \rightarrow 0} \frac{1}{\pi} \int_{|x-t|>\varepsilon} \frac{f(t)}{x-t} d t, \quad x \in \mathbb{R} .
$$

A question arises immediately concerning the existence of $\mathcal{H} f$, and we note that it was shown independently by Besicovitch and Kolmogoroff in the 1920 s that $\mathcal{H} f$ is finite a.e. in $\mathbb{R}$. It is also natural to ask whether, or to what extent, the operator $f \rightarrow \mathcal{H} f$ preserves properties of $f$, such as continuity, and it is this question we are concerned with here. A well-known result of Privalov (see [14, p. 121]) asserts that, if $f \in \operatorname{Lip}_{\alpha}(c, d)$, i.e. $f(x+t)-f(x)=O\left(|t|^{\alpha}\right)$ uniformly in $(c, d)$, and $0<\alpha<1$, then $\mathcal{H} f \in \operatorname{Lip}_{\alpha}(c, d)$ also, but in general the Hilbert transform of a continuous function need not be continuous. The transform of a continuous function does, however, show 'traces of continuity', in that $\mathcal{H} f$ has the intermediate-value property in the set of points $F$ for which it is finite ([14, p. 265]). These results for $\mathcal{H} f$ are usually proved for the conjugate function

$$
\tilde{f}(x)=\lim _{\eta \rightarrow 0} \frac{1}{2 \pi} \int_{\eta \leq|t-x| \leq \pi} f(t) \cot \frac{x-t}{2} d t,
$$

2000 Mathematics Subject Classification. 44A15, 42A50, 26B30, 31C15.

Key words and phrases. Hilbert transform, functions of bounded variation, exceptional sets, fine continuity. 
in which context $f$ is assumed to be a $2 \pi$-periodic function, but it is familiar that $\mathcal{H} f$ and $\tilde{f}$ have the same behaviour as a consequence of the fact that $1 / x-\cot x$ has a continuous extension to a neighbourhood of 0 .

In this note we consider the Hilbert transform of a function $f$ in $L(\mathbb{R})$ which has bounded variation in a finite interval $[c, d]$, so that $f$ has at most a countable set of points of discontinuity in $[c, d]$, and we describe certain continuity-type properties possessed by such transforms. More specifically, we show that, except for exceptional sets of $a \in(c, d)$ of capacity zero, $\mathcal{H} f(a)$ is finite and

$$
\lim _{x \rightarrow a, x \notin E} \mathcal{H} f(x)=\mathcal{H} f(a),
$$

where the excluded set $E$ is metrically 'thin' at $a$, when measured in terms of an appropriate capacity. Results of this type, when applied to the conjugate function, lead to theorems involving the tangential boundary behaviour of analytic functions (see [6], [11], or [13], for example).

\section{CAPACity}

The capacities we use to measure the size of exceptional sets are classical and involve the Bessel kernels $G_{\alpha}$. An explicit integral formula for $G_{\alpha}, 0<\alpha \leq 1$, may be found in [2, p. 10], but for our purposes here it is enough to observe that $G_{\alpha}$ is an even, positive, and unbounded function in $L(\mathbb{R})$ which is decreasing in $(0, \infty)$, decays exponentially as $|x| \rightarrow \infty$, and satisfies

$$
\begin{gathered}
G_{\alpha}(x) \simeq|x|^{\alpha-1}, \quad 0<\alpha<1, \\
G_{1}(x) \simeq \log \frac{1}{|x|},
\end{gathered}
$$

where $u \simeq v$ means that $u / v$ is bounded above and below by positive constants for all sufficiently small non-zero $|x|$.

Definition 1. For a Borel set $E$ and $0<\alpha \leq 1$, we define

$$
C_{\alpha}(E)=\inf \{\mu(\mathbb{R})\},
$$

where the infimum is taken over all non-negative Radon measures $\mu$ for which

$$
\int_{\mathbb{R}} G_{\alpha}(x-t) d \mu(t) \geq 1 \quad \text { for all } x \in E
$$


Equivalently [5, p. 20],

$$
C_{\alpha}(E)=\sup \{\mu(E)\},
$$

where the supremum is taken over all measures $\mu \in \mathcal{M}^{+}(E)$, the class of non-negative Radon measures $\mu$ on $\mathbb{R}$ with support on $E$, for which

$$
\int_{\mathbb{R}} G_{\alpha}(x-t) d \mu(t) \leq 1 \quad \text { for all } x \in E .
$$

We say that $E$ has $\alpha$-capacity zero if $C_{\alpha}(E)=0$. Any set of $\alpha$ capacity zero has Lebesgue measure zero, but the converse is false in general. Also, $C_{\alpha}(E)=0$ implies $C_{\beta}(E)=0$ for $0<\beta<\alpha \leq 1$. We shall use the term logarithmic capacity for the case $\alpha=1$ of $\alpha$-capacity. Logarithmic capacity is often defined differently in different contexts, but the definition given above, based on a classical approach, is suitable for the results under discussion here, and, as noted below, it yields a capacity that is comparable to a standard Bessel capacity from $L^{p}$-capacity theory [7].

For $0<\alpha \leq 1$, we shall say that a property that holds true for all $x \in(c, d) \backslash E$, where $E$ has $\alpha$-capacity zero, is true $\alpha$-quasieverywhere in the interval $(c, d)$. For the case $\alpha=1$ we shall usually simply write quasi-everywhere.

As an illustration of how sparse the points of a set of zero logarithmic capacity are, we note that if $S$ is a Cantor set constructed in such a way that the set $S_{n}$ obtained at the $n$th step consists of the union of $2^{n}$ disjoint intervals, each of length $l_{n}$, then $S=\bigcap_{1}^{\infty} S_{n}$ has zero logarithmic capacity if and only if $\sum_{1}^{\infty} 2^{-n} \log \left(1 / l_{n}\right)=\infty$. (See [5, p. 31] or [2, Theorem 5.3.2].)

We derive next an elementary lower estimate for logarithmic capacity in terms of Lebesgue measure $m$. This lemma is a special case of a general result involving estimates of Bessel capacities in terms of Hausdorff measures (see [2, p. 139]), but we include a simple proof for the sake of completeness.

Lemma 1. Suppose that $E \subset \mathbb{R}$ and that $0<m(E)<1 / 2$. Then

$$
\frac{A}{\log \frac{1}{m(E)}} \leq C_{1}(E) .
$$

Here and below, $A$ denotes a positive absolute constant, but not necessarily the same one at each occurrence. 
Proof of Lemma 1. We define a measure $\mu \in \mathcal{M}^{+}(E)$ by setting $d \mu(t)=\beta \chi_{E}(t) d t$, where $\chi_{E}$ is the characteristic function of $E$ and $\beta$ is a positive constant that remains to be chosen. Then, for $x \in \mathbb{R}$, by the monotonicity of $G_{1}$,

$$
\begin{aligned}
\int_{\mathbb{R}} G_{1}(x-t) d \mu(t) & =\beta \int_{E} G_{1}(x-t) d t \\
& \leq \beta \int_{-m(E)}^{m(E)} G_{1}(t) d t \\
& \leq A \beta \int_{-m(E)}^{m(E)} \log \frac{1}{|t|} d t \leq A \beta m(E) \log \frac{1}{m(E)}
\end{aligned}
$$

If we now choose $\beta$ so that the last quantity equals 1 , then

$$
\int_{\mathbb{R}} G_{1}(x-t) d \mu(t) \leq 1 \quad \text { and } \quad \mu(E)=A / \log \frac{1}{m(E)} .
$$

The required result follows from (the second part of) Definition 1.

Remark. If $E=(-\delta, \delta)$ then $C_{1}(E) \simeq 1 /\left(\log \frac{1}{\delta}\right)$ as $\delta \rightarrow 0$, see $[2$, p. 131].

\section{Thin Sets and Fine Continuity}

The notions of thin sets and fine continuity are generalisations of ideas from classical potential theory. (For this classical theory, see Armitage and Gardiner [1].) We base our definitions on those of Meyers [7] and Adams and Hedberg [2, Chapter 6]. These authors work with different capacities, particularly the Bessel capacities $C_{\alpha, p}([7])$, but noting that ([4, Corollary 2.2])

$$
C_{1}(E) \leq C_{1 / 2,2}(E) \leq A C_{1}(E),
$$

it is readily seen that the case $\alpha=1$ of the following definition is a special case of the corresponding definitions in [8] and [2].

Definition 2. Suppose that $S \subset \mathbb{R}$ is a Borel set and that $0<\alpha \leq 1$. Then $S$ is said to be $\alpha$-logarithmically thin at $a \in(c, d)$, abbreviated to $\alpha$-thin at $a$, if

$$
\int_{0}^{t_{0}} C_{1}(S \cap(a-t, a+t)) \frac{d t}{t^{2-\alpha}}<\infty
$$


for some $t_{0}>0$. We say that a function $h:[c, d] \rightarrow \mathbb{R}$ is $\alpha$-finely continuous at $a$ if there is a set $S \subset \mathbb{R}$ such that $S$ is $\alpha$-thin at $a$ and

$$
\lim _{x \rightarrow a, x \in(c, d) \backslash S} h(x)=h(a) .
$$

Equivalently, see [2, Proposition 6.4.3], $h$ is $\alpha$-finely continuous at $a$ if

$$
\{x: x \in(c, d),|h(x)-h(a)| \geq \varepsilon\}
$$

is $\alpha$-thin at $a$ for all $\varepsilon>0$.

When $\alpha=1$, we shall write thin and finely continuous for $\alpha$-thin and $\alpha$-finely continuous, respectively. We note that thin and finely continuous are then equivalent to the concepts of $(1 / 2,2)$-thin and $(1 / 2,2)$-finely continuous (with $N=1$ ) in [2, Chapter 6].

If a set $S$ is thin at $a$, then, for every fixed $\lambda>1$,

$$
m(S \cap(a-t, a+t))=O\left(t^{\lambda}\right) \quad \text { as } \quad t \rightarrow 0 .
$$

To see that this is a consequence of (3.1) with $\alpha=1$, note first that, for $t \in\left(0, t_{0}^{2}\right)$,

$$
\frac{1}{2} C_{1}(S(t)) \log \frac{1}{t}=C_{1}(S(t)) \int_{t}^{t^{1 / 2}} \frac{d r}{r} \leq \int_{t}^{t^{1 / 2}} \frac{C_{1}(S(r))}{r} d r \rightarrow 0
$$

as $t \rightarrow 0$, where we have written $S(t)$ for $S \cap(a-t, a+t)$. Hence

$$
C_{1}(S(t))=o\left(\left(\log \frac{1}{t}\right)^{-1}\right) \text { as } t \rightarrow 0 .
$$

It is a simple matter to show, using (2.2), that (3.2) follows from this if $m(S(t))>0$ for $t>0$. If $m\left(S\left(t_{1}\right)\right)=0$ for some $t_{1}>0$, then $m(S(t))=0$ for $0<t \leq t_{1}$, and (3.2) is trivially true. A similar argument to the above shows that (3.1), with $0<\alpha<1$, implies that $C_{1}(S(t))=o\left(t^{1-\alpha}\right)$ and hence, using (2.2) again, that

$$
m(S \cap(a-t, a+t))=O\left(\exp \left(-\frac{B}{t^{1-\alpha}}\right)\right) \quad \text { as } \quad t \rightarrow 0
$$

for every positive constant $B$. 


\section{Fine Continuity of the Hilbert Transform}

Let $[c, d]$ be a finite closed interval and let $\chi \equiv \chi_{[c, d]}$ denote the characteristic function of $[c, d]$. Suppose $x \in(c, d)$ and set $\delta(x)=$ $\min \{d-x, x-c\}>0$. Then

$$
\begin{aligned}
\pi \mathcal{H} f(x) & =\lim _{\varepsilon \rightarrow 0} \int_{|x-t|>\varepsilon} \frac{f(t) \chi(t)}{x-t} d t+\int_{|x-t|>\delta(x)} \frac{f(t)(1-\chi(t))}{x-t} d t \\
& =\pi \mathcal{H} f \chi(x)+g(x)
\end{aligned}
$$

for $x \in(c, d)$. We show that $g$ is continuous in $(c, d)$. To this end, let $a \in(c, d)$ and write $\delta$ for $\delta(a)$. Then, if $t \in \mathbb{R} \backslash[c, d]$ and $|x-a|<\delta / 2$, we have $|x-t| \geq \delta / 2$ and

$$
\left|\frac{f(t)(1-\chi(t))}{x-t}\right| \leq \frac{2|f(t)|}{\delta} .
$$

The continuity of $g$ at $a$, and hence in $(c, d)$, now follows from the Lebesgue dominated convergence theorem.

Suppose next that $f \in B V[c, d]$, i.e. that $f$ has bounded variation on $[c, d]$. Then, by integration by parts, for $x \in(c, d)$ and $\varepsilon<\delta(x)$,

$$
\begin{aligned}
\int_{|x-t|>\epsilon} \frac{f(t) \chi(t)}{x-t} d t= & \int_{[c, d] \backslash(x-\varepsilon, x+\varepsilon)} \frac{f(t)}{x-t} d t \\
= & f(c) \log (x-c)-f(d) \log (d-x) \\
& +\{f(x+\varepsilon)-f(x-\varepsilon)\} \log \varepsilon \\
& +\int_{[c, d] \backslash(x-\varepsilon, x+\varepsilon)}^{\log |x-t| d f(t) .}
\end{aligned}
$$

We now state a lemma on monotonic functions that we need.

Lemma 2. ([12, Lemma 1]; see also [10, Theorem VII]) Suppose that $F$ is increasing on $[c, d]$ and extended to $\mathbb{R}$ by setting $F(x)=$ $F(d)$ for $x>d$ and $F(x)=F(c)$ for $x<c$. Then

$$
\int_{c}^{d} \log |x-t| d F(t)
$$

is finite quasi-everywhere in $[c, d]$. If $x=a \in(c, d)$ is a value for which (4.2) is finite, then

$$
F(a+\delta)-F(a-\delta)=o\left(1 / \log \frac{1}{\delta}\right), \quad \delta \rightarrow 0
$$


and

$$
\int_{0}^{1} \frac{F(a+t)-F(a-t)}{t} d t<\infty .
$$

Furthermore, for $0<\alpha<1$, we have $([12$, p. 452])

$$
\int_{0}^{1} \frac{F(x+t)-F(x-t)}{t^{2-\alpha}} d t<\infty
$$

for all $x \in(c, d)$, except possibly for a set of $x$ of $\alpha$-capacity zero.

Remark. An easy consequence of (4.5) and the monotonicity of $F$ is that, for $0<\alpha<1$,

$$
F(x+\delta)-F(x-\delta)=o\left(\delta^{1-\alpha}\right), \quad \delta \rightarrow 0,
$$

$\alpha$-quasi-everywhere in $[c, d]$. The relations (4.3) and (4.6), together with their associated exceptional sets, are intermediate results between two familiar facts for monotonic functions, namely that such functions are continuous outside a countable set and differentiable outside a set of Lebesgue measure zero.

Since $f \in B V[c, d]$, there are increasing functions $F_{1}, F_{2}$ for which $f=F_{1}-F_{2}$ on $[c, d]$. It thus follows from Lemma 2 and (4.1), since $C_{1}\left(E_{1} \cup E_{2}\right)=0$ if $C_{1}\left(E_{1}\right)=C_{1}\left(E_{2}\right)=0$, that

$$
\begin{aligned}
\pi \mathcal{H} f \chi(x) & =\lim _{\epsilon \rightarrow 0} \int_{|x-t|>\epsilon} \frac{f(t) \chi(t)}{x-t} d t \\
& =f(c) \log (x-c)-f(d) \log (d-x)+\int_{c}^{d} \log |x-t| d f(t)
\end{aligned}
$$

is finite quasi-everywhere in $(c, d)$.

We now state our main theorem.

Theorem 1. If $f \in L(\mathbb{R})$ and $f \in B V[c, d]$, then $\mathcal{H} f$ is $\alpha$-finely continuous $\alpha$-quasi-everywhere in $[c, d]$, where $0<\alpha \leq 1$.

As noted above, $\mathcal{H} f=\mathcal{H} f \chi+g$, where $g$ is continuous in $(c, d)$, so, by (4.7), Theorem 1 will follow once we prove the following result.

Theorem 2. Suppose that $F$ is increasing on $[c, d]$ and extended to $\mathbb{R}$ as in Lemma 2. Set

$$
h(x)=\int_{c}^{d} \log \frac{1}{|x-t|} d F(t), \quad x \in[c, d] .
$$


Suppose that $a \in(c, d)$ and that $h(a)$ is finite. Then $h$ is finely continuous at a. Furthermore, for $0<\alpha<1$, the logarithmic potential $h$ is $\alpha$-finely continuous $\alpha$-quasi-everywhere in $[c, d]$.

Remarks. 1. The case $\alpha=1$ of Theorem 1 follows from the first part of Theorem 2 since, by Lemma $2, h$ is finite quasi-everywhere in $[c, d]$. This particular result concerning the logarithmic potential is known and is a consequence of standard results in potential theory (see [9] for an explicit formulation and an alternative approach to the one outlined here).

2. If $h$ is finely continuous at $a$, then

$$
\lim _{x \rightarrow a, x \notin E} h(x)=h(a),
$$

where $E$ is thin at $a$ and consequently, by the remarks at the end of Section 3

$$
m(E \cap(a-\delta, a+\delta))=O\left(\delta^{\lambda}\right), \quad \delta \rightarrow 0,
$$

for every $\lambda>1$. It follows that the result for the case $\alpha=1$ in Theorem 1 here, when stated in terms of the conjugate function $\tilde{f}$ (see the Introduction above), sharpens the conclusion of [13, Theorem 1].

Proof of Theorem 2. We begin with the proof of the first part of the theorem. Suppose that $h(a)$ is finite where $a \in(c, d)$. Let $\varepsilon>0$ be given and set

$$
E(\varepsilon)=\{x \in[c, d]:|h(x)-h(a)| \geq \varepsilon\} .
$$

It is enough, by (the second part of) Definition 2, to prove that $E(\varepsilon)$ is thin at $a$. We assume, as we may, that $d-c \leq 1$, so that the integrand in (4.8) is non-negative. By Fatou's lemma,

$$
\begin{aligned}
\liminf _{x \rightarrow a} h(x) & =\liminf _{x \rightarrow a} \int_{c}^{d} \log \frac{1}{|x-t|} d F(t) \\
& \geq \int_{c}^{d} \liminf _{x \rightarrow a} \log \frac{1}{|x-t|} d F(t)=h(a),
\end{aligned}
$$

and it follows that

$$
\liminf _{x \rightarrow a, x \in E(\varepsilon)} h(x) \geq h(a)+\varepsilon .
$$

We show next that, for all sufficiently small $\rho$,

$$
C_{1}(E(\varepsilon) \cap B(a, \rho)) \leq \frac{A}{\varepsilon}[F(a+2 \rho)-F(a-2 \rho)],
$$


where $B(a, \rho)=(a-\rho, a+\rho)$ for $\rho>0$. The short argument we use to do this is a simple adaptation of the argument used in [2, p. 180] to derive an analogous inequality for potentials of $L^{p}$ functions.

We begin by setting

$$
h_{r}(x)=\int_{t \in B(a, r)} \log \frac{1}{|x-t|} d F(t), \quad x \in[c, d],
$$

where $r \in(0,1 / 2)$ is chosen so that $B(a, r) \subset[c, d]$ and

$$
h_{r}(a) \leq \frac{\varepsilon}{4} .
$$

Such a choice of $r$ is possible by the finiteness of $h(a)$. We note that, since

$$
\lim _{x \rightarrow a} \int_{B^{\prime}(a, r)} \log \frac{1}{|x-t|} d F(t)=\int_{B^{\prime}(a, r)} \log \frac{1}{|a-t|} d F(t),
$$

where $B^{\prime}(a, r)=[c, d] \backslash B(a, r)$, it follows from (4.9) that

$$
\liminf _{x \rightarrow a, x \in E(\varepsilon)} h_{r}(x) \geq h_{r}(a)+\varepsilon .
$$

Let $0<\rho \leq r$. Then, if $|x-a| \leq \rho / 2$ and $|t-a| \geq \rho$, we have $|t-x| \geq|t-a| / 2$, and so, using (4.11),

$$
\begin{aligned}
\int_{B(a, r) \backslash B(a, \rho)} \log \frac{1}{|x-t|} d F(t) & \leq \int_{B(a, r)} \log \frac{2}{|a-t|} d F(t) \\
& \leq 2 h_{r}(a) \leq \frac{\varepsilon}{2}
\end{aligned}
$$

We now choose $\rho_{0} \in(0, r)$ such that

$$
h_{r}(x) \geq \frac{3 \varepsilon}{4}
$$

for $|x-a| \leq \rho_{0}$ and $x \in E(\varepsilon)$. Then, for $0<\rho<\rho_{0}$ and $x \in$ $E(\varepsilon) \cap B\left(a, \frac{\rho}{2}\right)$,

$$
\int_{B(a, \rho)} \log \frac{1}{|x-t|} d F(t)=\int_{B(a, r)}-\int_{B(a, r) \backslash B(a, \rho)} \geq \frac{\varepsilon}{4},
$$

that is,

$$
\int_{\mathbb{R}} \log \frac{1}{|x-t|} d \mu(t) \geq 1, \quad x \in E(\varepsilon) \cap B\left(a, \frac{\rho}{2}\right),
$$

where $d \mu(t)=(4 / \varepsilon) \chi_{B(a, \rho)}(t) d F(t)$. Since, by $(2.1)$,

$$
\log (1 /|x-t|) \leq A G_{1}(x-t)
$$


for $x, t \in[c, d]$, it follows from (the first part of) Definition 1, that $C_{1}\left(E(\varepsilon) \cap B\left(a, \frac{\rho}{2}\right)\right) \leq A \mu(B(a, \rho)) \leq \frac{4 A}{\varepsilon}[F(a+\rho)-F(a-\rho)]$, and we have established (4.10). From (4.4) we now easily obtain

$$
\int_{0}^{1} \frac{1}{t} C_{1}(E(\varepsilon) \cap B(a, t)) d t<\infty
$$

so $E(\varepsilon)$ is thin at $a$. This proves the first part of Theorem 2. The case $0<\alpha<1$ follows from (4.10) and the last statement of Lemma 2 . This completes the proof of Theorem 2 and hence the proof of Theorem 1 as well.

We conclude with an observation relating to Theorem 1 . The exceptional set of $a \in(c, d)$ in Theorem 1 associated with a value of $\alpha \in(0,1)$ is, in general, larger than the exceptional set corresponding to $\alpha=1$, since $C_{1}(E)=0$ implies $C_{\alpha}(E)=0$ and the converse is false, but the excluded set $E$ at $a$ for which

$$
\lim _{x \rightarrow a, x \notin E} \mathcal{H} f(x)=\mathcal{H} f(a)
$$

is smaller, as indicated by a comparison between (3.2) and (3.3) with $S$ replaced by $E$.

\section{REFERENCES}

[1] D. H. Armitage and S. J. Gardiner, Classical potential theory, SpringerVerlag, London, 2001.

[2] D. R. Adams and L. I. Hedberg, Function spaces and potential theory, Springer-Verlag, Berlin, 1996.

[3] D. R. Adams and N. G. Meyers, Thinness and Wiener criteria for non-linear potentials, Indiana Univ. Math. J. 22 (1972), 169-197.

[4] D. R. Adams and N. G. Meyers, Bessel potentials. Inclusion relations among classes of exceptional sets, Indiana Univ. Math. J. 22 (1973), 873-905.

[5] L. Carleson, Selected problems on exceptional sets, Van Nostrand, Princeton, New Jersey, 1967.

[6] J. L. Doob, The boundary values of analytic functions, Trans. Amer. Math. Soc. 34 (1932), 153-170.

[7] N. G. Meyers, A theory of capacities for potentials of functions in Lebesgue classes, Math. Scand. 26 (1970), 255-292.

[8] N. G. Meyers, Continuity properties of potentials, Duke Math. J. 42 (1975), $157-166$.

[9] Y. Mizuta, Potential theory in Euclidean spaces, Gakkōtosho Co. Ltd., Tokyo, 1996.

[10] R. Salem and A. Zygmund, Capacity of sets and Fourier series, Trans. Amer. Math. Soc. 97 (1946), 23-41. 
[11] U. V. Satyanarayana and M. L. Weiss, Tangential asymptotic values of bounded analytic functions, Proc. Amer. Math. Soc. 41 (1973), 167-172.

[12] J. B. Twomey, Tangential boundary behaviour of the Cauchy integral, J. London Math. Soc., (2) 37 (1988), 447-454.

[13] J. B. Twomey, Strong approximate continuity properties of certain conjugate functions, Math. Proc. Royal Irish Acad. 105A (1) (2005), 49-58.

[14] A. Zygmund, Trigonometric series, 2nd ed., vol. 1, Cambridge Univ. Press, New York, 1959.

J. B. Twomey,

Department of Mathematics,

University College Cork,

Cork, Ireland

b.twomey@ucc.ie

Received on 28 March 2006 and in revised form on 18 December 2006. 